\title{
A Study of Mammographic Image Segmentation with its Morphological Operation
}

\author{
G Balanagireddy ${ }^{1}$, J K Periasamy ${ }^{2}$, G Saritha $^{3}$, M Sujatha ${ }^{4}$, G Nirmalapriya ${ }^{5}$ \\ \{nagireddy.nano@gmail.com¹,jkperiasamy.cse@sairam.edu.in², saritha.ganesan@gmail.com3, \\ sujakarthik77@kluniversity.in ${ }^{4}$,nirmalapriya.g@ritchennai.edu.in ${ }^{5}$ \}
}

\begin{abstract}
${ }^{1}$ Assistant Professor, Department of Electronics and Communication Engineering, PACE Institute of Technology and Sciences, Ongole, Andhra Pradesh, India., ${ }^{2}$ Associate Professor, Department of Computer Science and Engineering, Sri Sairam Engineering College, Chennai, Tamil Nadu, India. ${ }^{3}$ Assistant Professor, Department of Electronics and Communication Engineering, Sri Sairam Institute of Technology, Chennai, Tamil Nadu, India, ${ }^{4}$ Professor, Department of Electronics and Communication Engineering, Koneru Lakshmaiah Education Foundation, Vijayawada, Andhra Pradesh, India, ${ }^{5}$ Professor, Department of Electronics and Communication Engineering, Rajalakshmi Institute of Technology, Chennai, Tamil Nadu, India.
\end{abstract}

\begin{abstract}
The Malign cell extraction and segmentation differentiation from normal cells is widely researched topic. The process of segmentation with single strategy might miss the features leading to increased mortality rate. This work characterizes the different segmentation methods and two simulation tools for mammogram images. The non-feature pixel values are represented by the nearest feature pixel in distance by watershed segmentation. Simulations are performed with ImageJ using Morphological library where binary mammogram images are analysed with connected components and distance based watershed transform. Finally the mammogram image in DICOM format is analysed for segmenting spanning lower and upper threshold with clustering..
\end{abstract}

Keywords: Breast cancer, Image segmentation and Morphological operations.

\section{Introduction}

The process variations in malignant cells spanning across varying size is observed in medical images to provide appropriate diagnosis. Digital mammogram has been the widely imaging modality uses fifty two percentages whereas the other modules occupy the remaining forty eight percentages [6]. The step by step process from imaging module with early detection to subsequent segmentation, classification is vital to reduce call back and mortality rates. ImageJ tool is used with morphological library work [11] with electronic samples of the binary image. Segmentation of non significant minima of tumour areas is clearly segmented.

Semiautomatic segmentation has been used where there is a failure in accuracy of fully automated segmentation using ITK SNAP [12]. In this work malign images with annotation has been used thus threshold and clustering of images is performed with ITK SNAP using Segment 3D. The paper is organised as follows. The section 2 reviews the limitations of mammogram images with discussion on machine learning, segmentation and describes the associated problems. Section 3 deals with "ImageJ" and ITK SNAP procedure for the underlying segmentation works. Section 4 provides results and discussion using ImageJ. Section 5 concludes the overall work and future direction.

\section{Literature review}

The machine learning algorithms predominantly use training followed by testing from the same feature space of cohort of images. The mammogram approach which underlies machine learning fails when the distribution of feature space varies across the newly procured images. Transfer learning approaches are suggested superior performance in [1] across several mammogram databases improves classification accuracy and reducing the computation time in processing new models.

\subsection{Image segmentation}

The contrast of mammogram images using "linearly quantile separation and histogram equalization" prior to segmentation improves the accuracy and reduces the false alarm rate. The simulation has been carried out using "LQSHE-GRA" which is suitable for MIAS database and unsupervised segmentation is difficult [2]. Selecting a best segmentation process by comparing it to a best atlas image has been discussed. The process incurs demon registration where in a static image and a moving image has been used for interpreting the nature 
of deformation [3]. Multilevel segmentation along with "Harris Hawk optimization" has been incorporated in digital mammograms. The superiorly performance of image segmentation is achieved using multi-level segmentation. This is accomplished via a fitness function using "Minimum cost entropy Thresholding" [4]. Inhomogenous region across the tumor area has been worked with active contour methods considering the local and global regions. Energy function along with Laplace function eliminates the false contour [5]. Generative Adversarial Network (GAN) has been discussed in [7] wherein the binary mask is generated and compared with ground truth. Further the losses are combined and feedback to generator network to minimize the losses. The shape classification along with molecular subtypes is also studied in GAN.

The outlier detection in noisy mammogram images for maintaining consistency has been done using "Intuitionistic Possibilitic Fuzzy C-mean Clustering" (IPFCM). The superior result of classification has been achieved even in the case of increasing noise in IPFCM [8].However, assigning member ship functions for Intuitionistic may fail to work for all images in minimal execution time.

\section{Proposed work}

The ImageJ procedure has been given for connected components with distance based watershed is shown from step 1 to step 5 .

Step1. The given image from a DDSM database is taken.

Step2. The malign images with landmarks are considered for processing.

Step3. Image is being converted into a binary format.

Step4. From the morphological library connected components has been used to calculate the distance map in binary image. The value of 4 has been used for analysis with 16 bits.

Step5. Then distance transform watershed segmentation has been done using "Borgefors transform" [10].

The ITK SNAP procedure has been given for Manual Thresholding and clustering is shown from step 1 to step 3.

Step1. The given image is converted into DICOM format.

Step2. Perform manual Thresholding of the given image to segment the tumour area using segment 3D option.

Step3. Perform clustering with appropriate cluster size and foreground cluster.

\section{Results and discussion}

The images has been obtained from DDSM [9] where in malignant samples are alone being analysed through ImageJ software. The first patient meta-details consist of age 72 with density 2 and malignancy is found in left breast shown in figure 2 . The right breast does not contain any malignancy.

\subsection{Results with Image J}
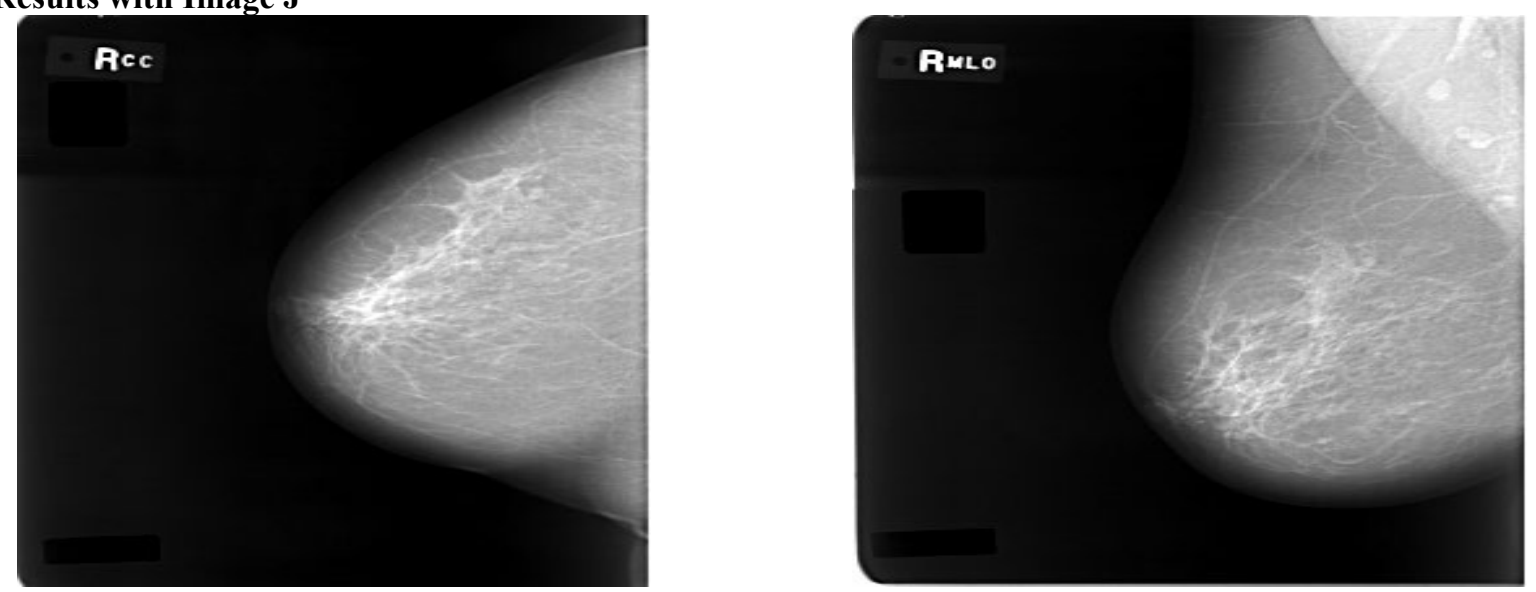

Figure 1(a) Shows the right CC view and 1(b) the right MLO view with no malign cells as landmarks. 

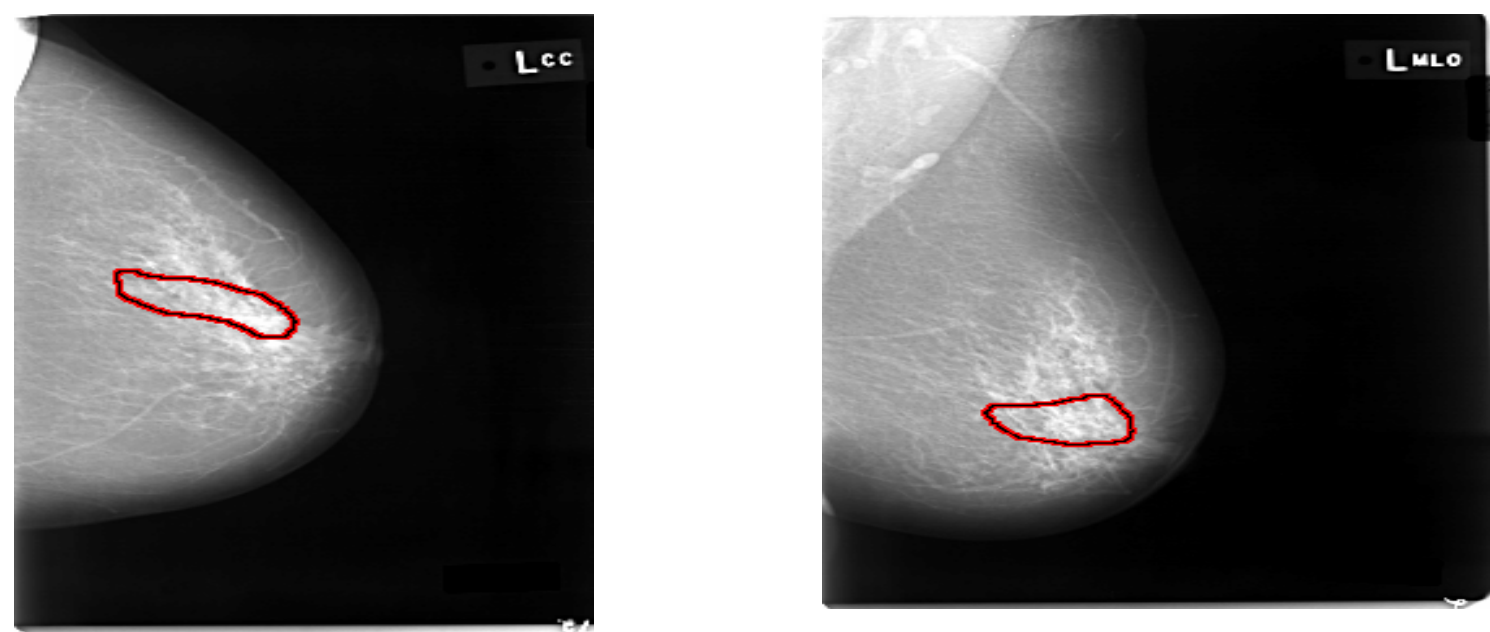

Figure 2 (a) Shows the left CC view and 2 (b) the left MLO view with malign cells as landmarks shown in red colour.
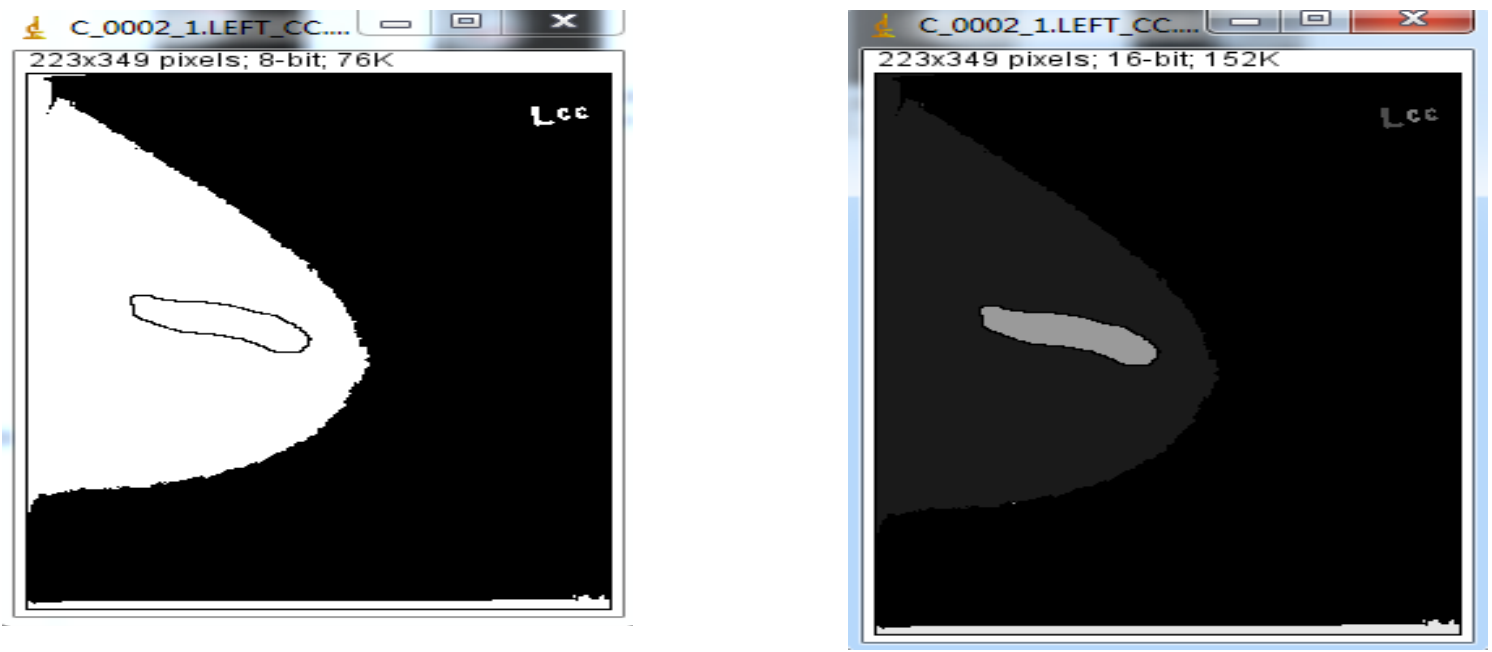

Figure 3(a) Shows the binary image in Left CC view. Figure 4 (b) Shows the binary image in connected component view.

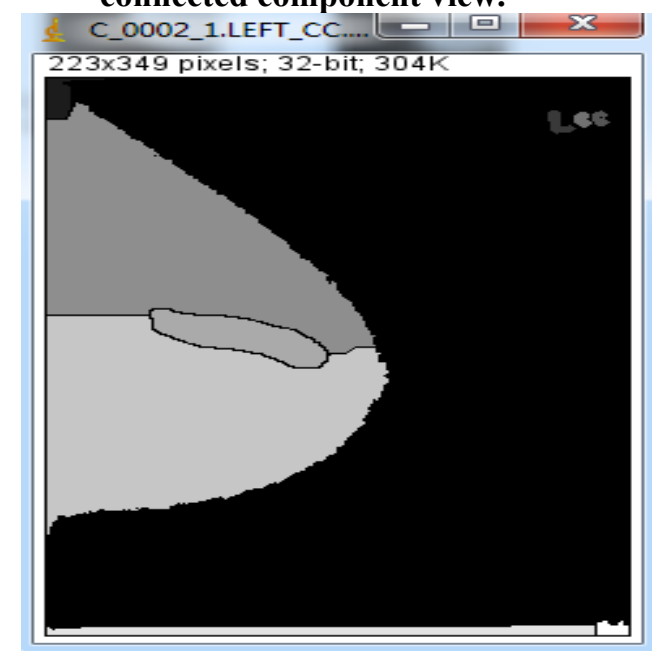

Figure 3(c) Distance transform watershed segmentation of Left CC view. 
I C_OOO2_1.LEFT_MLO_UPE:L

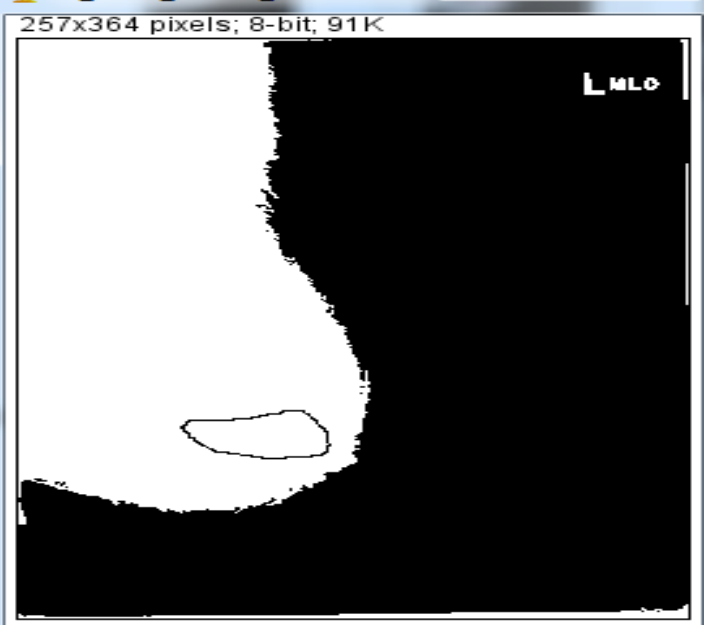

Figure 4(a) Shows the binary image in Left MLO view. Figure 4 (b) Shows the binary image in connected component view.

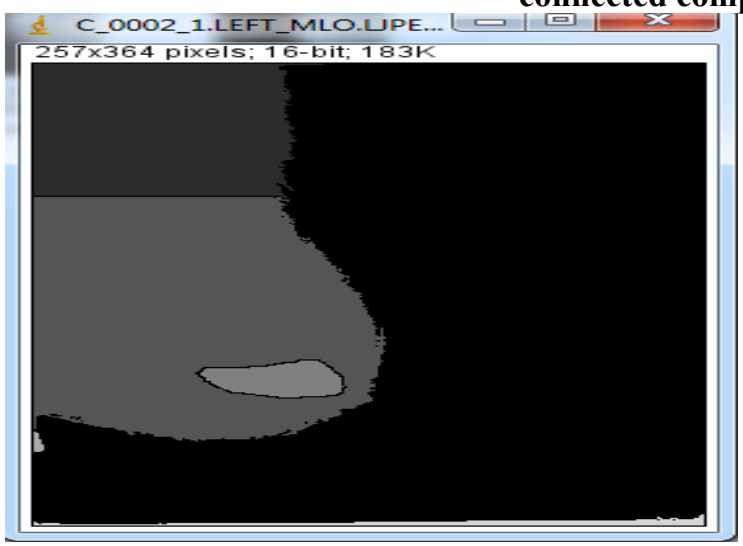

Figure 4(c) Distance transform watershed segmentation of Left MLO view.

\subsection{Results with ITK SNAP}

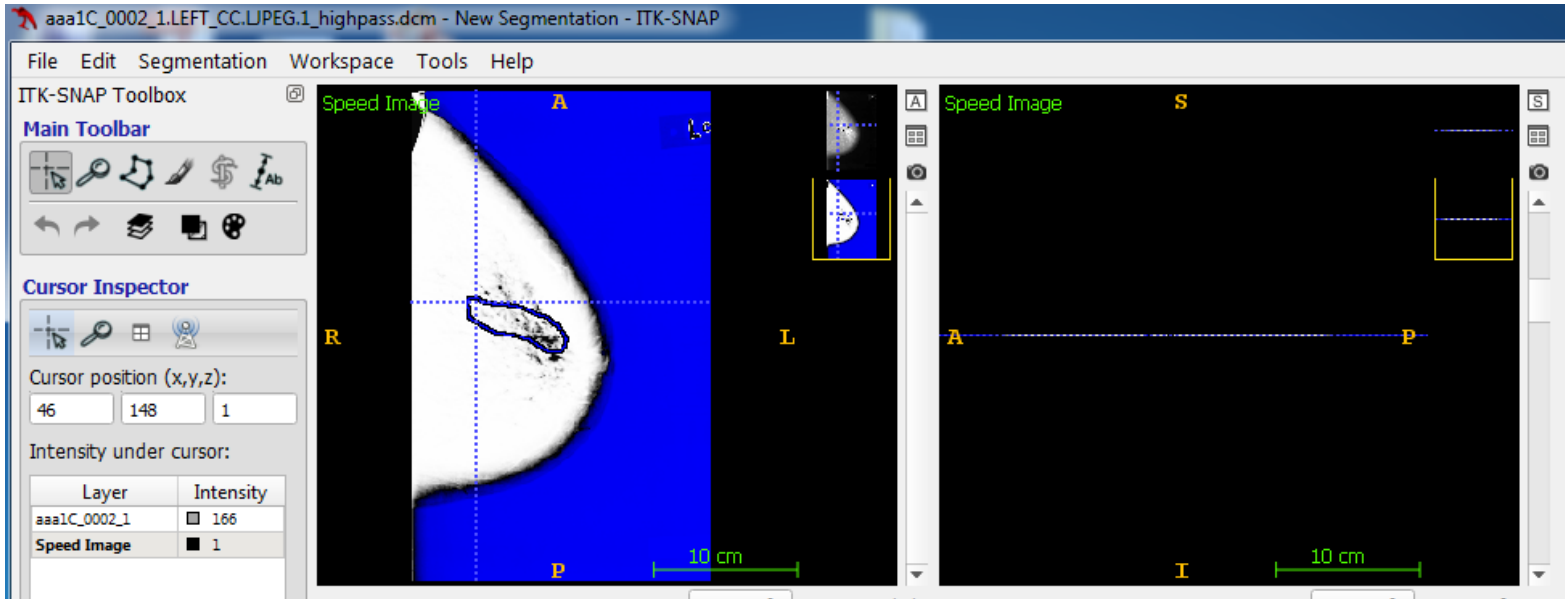

Figure 5a Thresholding image with $\mathbf{L}$ eft $\overline{\mathbf{C}} \ddot{\mathrm{C}}$ view using ITK snap tool. 


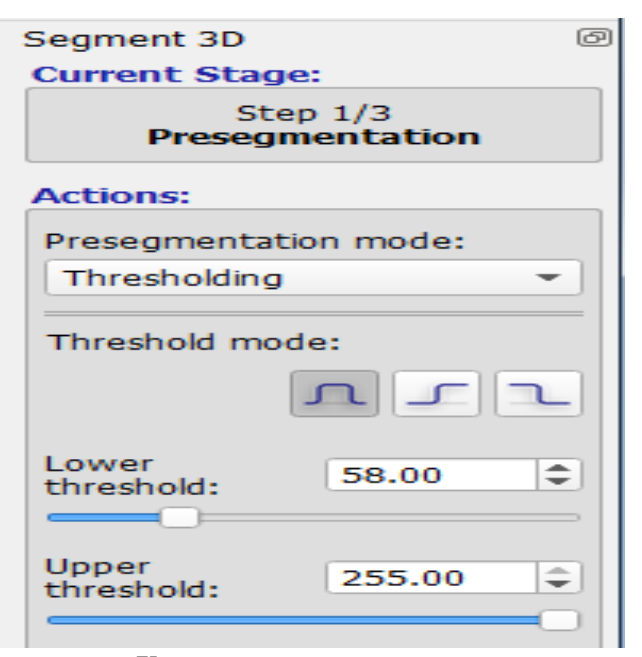

$5 b$

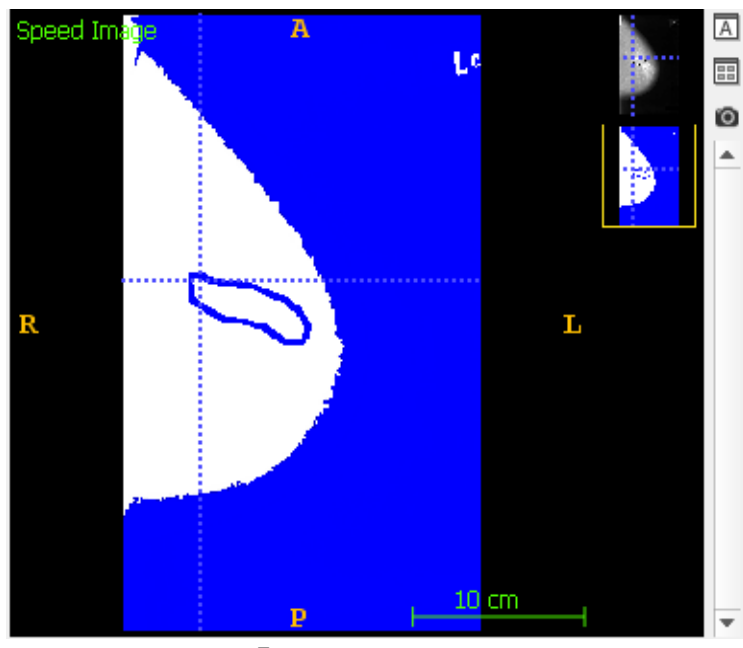

$5 c$

Figure 5b Corresponding values of lower threshold and upper threshold used for interpreting 5 a.

Figure 5c The final clustering image is shown.

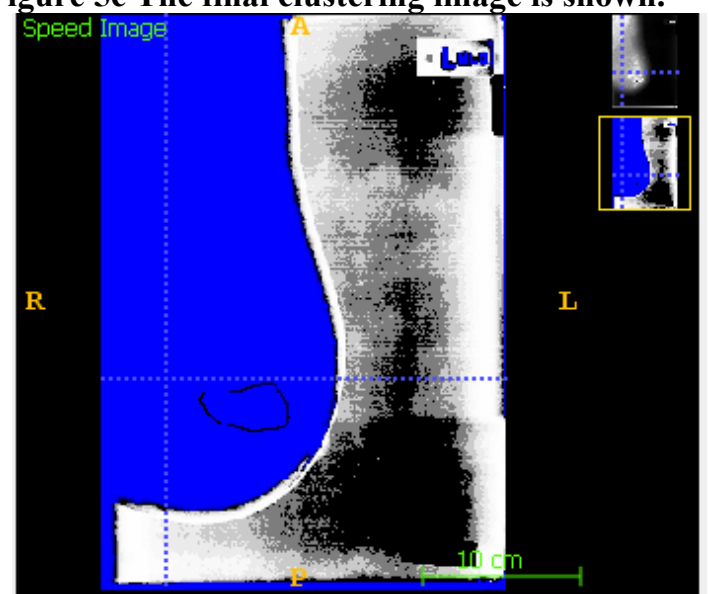

Figure 6a Thresholding image with Left MLO view using ITK snap tool.
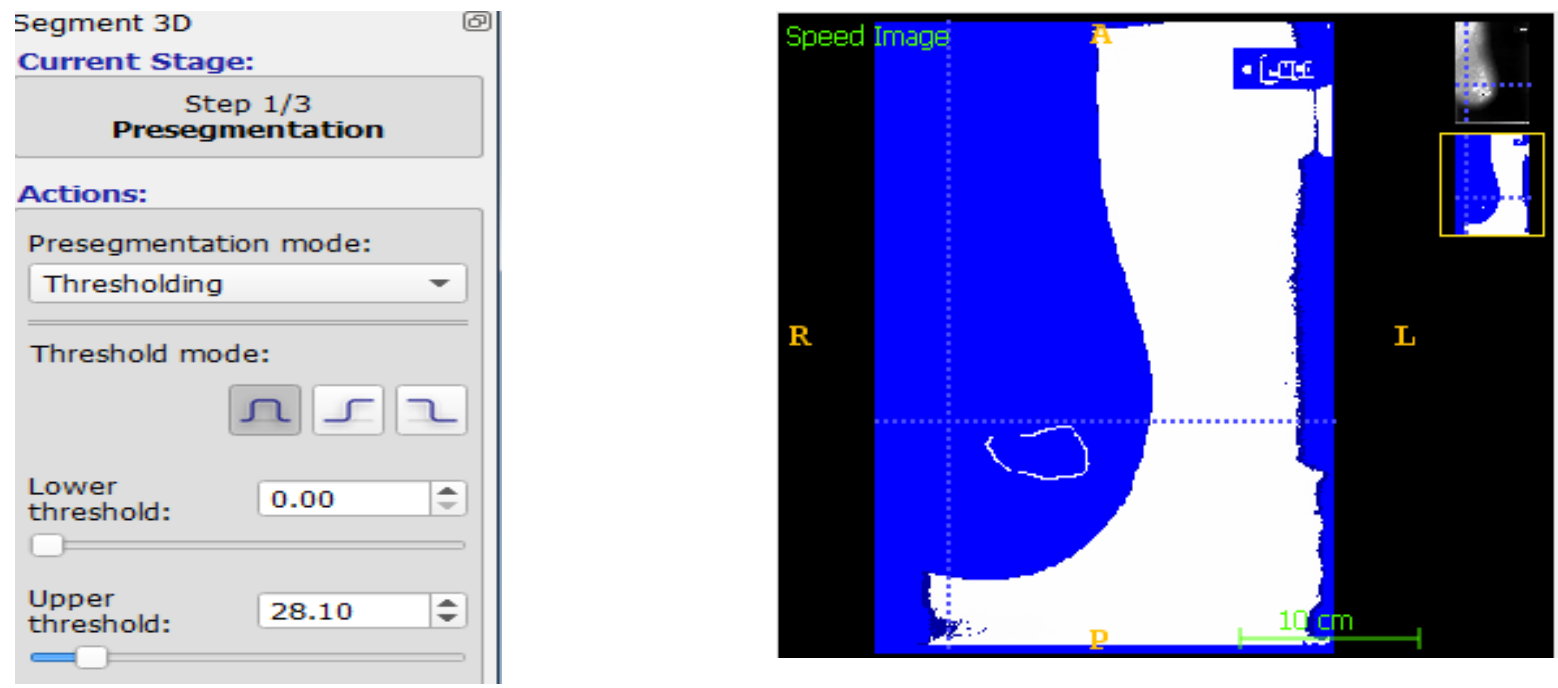

Figure 6b Corresponding values of lower threshold and upper threshold used for interpreting $6 \mathrm{a}$.

Figure $6 \mathrm{c}$ The final clustering image is shown. 


\section{Conclusion}

Two corresponding views of mammogram images with annotated landmarks have been taken for segmentation. The process is initially being analysed with connected pixels and distance based watershed transform using ImageJ with plug-in for performing morphological operation. The second half works with DICOM format in manual threshold and clustering to illustrate the spread of malign cells across the image with its corresponding orientation. Thus an elusive work customised to different segmentation has been done. Future work will deal with multimodal techniques with image fusion such as thermography and mammogram in identifying cancerous cells with its corresponding morphology.

\section{References}

[1] Kumar, D., Kumar, C., \& Shao, M. (2017, December). Cross-database mammographic image analysis through unsupervised domain adaptation. In 2017 IEEE International Conference on Big Data (Big Data) (pp. 4035-4042). IEEE.

[2] Gupta, B., Tiwari, M., \& Lamba, S. S. (2019). Visibility improvement and mass segmentation of mammogram images using quantile separated histogram equalisation with local contrast enhancement. CAAI Transactions on Intelligence Technology, 4(2), 73-79.

[3] Sharma, M. K., Jas, M., Karale, V., Sadhu, A., \& Mukhopadhyay, S. (2019). Mammogram segmentation using multi-atlas deformable registration. Computers in biology and medicine, 110, 244-253.

[4] Rodríguez-Esparza, Erick, Laura A. Zanella-Calzada, Diego Oliva, Ali Asghar Heidari, Daniel Zaldivar, Marco Pérez-Cisneros, and Loke Kok Foong. "An Efficient Harris Hawks-inspired Image Segmentation Method." Expert Systems with Applications (2020): 113428.

[5] Niaz, A., Memon, A. A., Rana, K., Joshi, A., Soomro, S., Kang, J. S., \& Choi, K. N. (2020). Inhomogeneous Image Segmentation Using Hybrid Active Contours Model With Application to Breast Tumor Detection. IEEE Access, 8, 186851-186861.

[6] Mahmood, T., Li, J., Pei, Y., Akhtar, F., Imran, A., \& Rehman, K. U. (2020). A Brief Survey on Breast Cancer Diagnostic With Deep Learning Schemes Using Multi-Image Modalities. IEEE Access, 8, 165779-165809.

[7] Singh, V. K., Rashwan, H. A., Romani, S., Akram, F., Pandey, N., Sarker, M. M. K., \& Torrents-Barrena, J. (2020). Breast tumor segmentation and shape classification in mammograms using generative adversarial and convolutional neural network. Expert Systems with Applications, 139, 112855.

[8] Chowdhary, C. L., Mittal, M., Pattanaik, P. A., \& Marszalek, Z. (2020). An efficient segmentation and classification system in medical images using intuitionist possibilistic fuzzy C-mean clustering and fuzzy SVM algorithm. Sensors, 20(14), 3903.

[9] http://www.eng.usf.edu/cvprg/Mammography/DDSM/thumbnails/cancers/cancer_01/case0002 /C-0002-1.html.

[10] Borgefors, G. (1986). Distance transformations in digital images. Computer vision, graphics, and image processing, 34(3), 344-371.

[11] Legland, D., Arganda-Carreras, I., \& Andrey, P. (2016). MorphoLibJ: integrated library and plugins for mathematical morphology with ImageJ. Bioinformatics, 32(22), 3532-3534.

[12] Yushkevich, P. A., Gao, Y., \& Gerig, G. (2016, August). ITK-SNAP: An interactive tool for semi-automatic segmentation of multi-modality biomedical images. In 2016 38th Annual International Conference of the IEEE Engineering in Medicine and Biology Society (EMBC) (pp. 3342-3345). IEEE. 